Supplement of Biogeosciences, 16, 2443-2465, 2019

https://doi.org/10.5194/bg-16-2443-2019-supplement

(C) Author(s) 2019. This work is distributed under

the Creative Commons Attribution 4.0 License.

(c) (1)

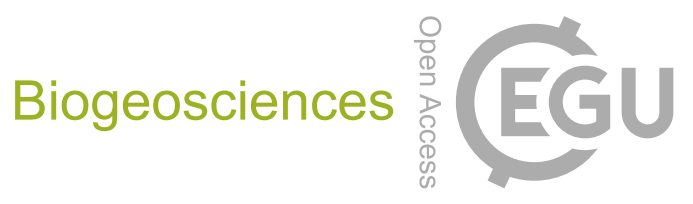

Supplement of

\title{
Organic signatures in Pleistocene cherts from Lake Magadi (Kenya) - im- plications for early Earth hydrothermal deposits
}

Manuel Reinhardt et al.

Correspondence to: Manuel Reinhardt (mreinha@gwdg.de)

The copyright of individual parts of the supplement might differ from the CC BY 4.0 License. 


\section{Interior-versus-exterior experiments (LM-1692 and LM-1695)}

Crushed and powdered surface cut-offs (exterior) and the inner blocks (interior) of LM-1692 and LM-1695 were extracted and derivatized using a similar protocol as described in Section 2.2. In brief, $10 \mathrm{~g}$ sample powder $(2 \times$ interior and $2 \times$ exterior per sample) was ultrasonically extracted with $20 \mathrm{~mL} \mathrm{DCM} / \mathrm{MeOH}(2 / 1, v / v)$, DCM / MeOH (3/1, v/v) and DCM (10 min,

5 respectively). All TOEs were desulfurized with reduced $\mathrm{Cu}$ and hydrolyzed using TMCS / $\mathrm{MeOH}\left(1 / 9, v / v\right.$; heated at $80{ }^{\circ} \mathrm{C}$ for $1 \mathrm{~h} 30 \mathrm{~min})$. Subsequently, the extracts were derivatized with BSTFA / pyridine $\left(3 / 2, v / v\right.$; heated at $40{ }^{\circ} \mathrm{C}$ for $\left.1 \mathrm{~h}\right)$. All samples were analyzed via GC-MS using the parameters described in Section 2.4. 


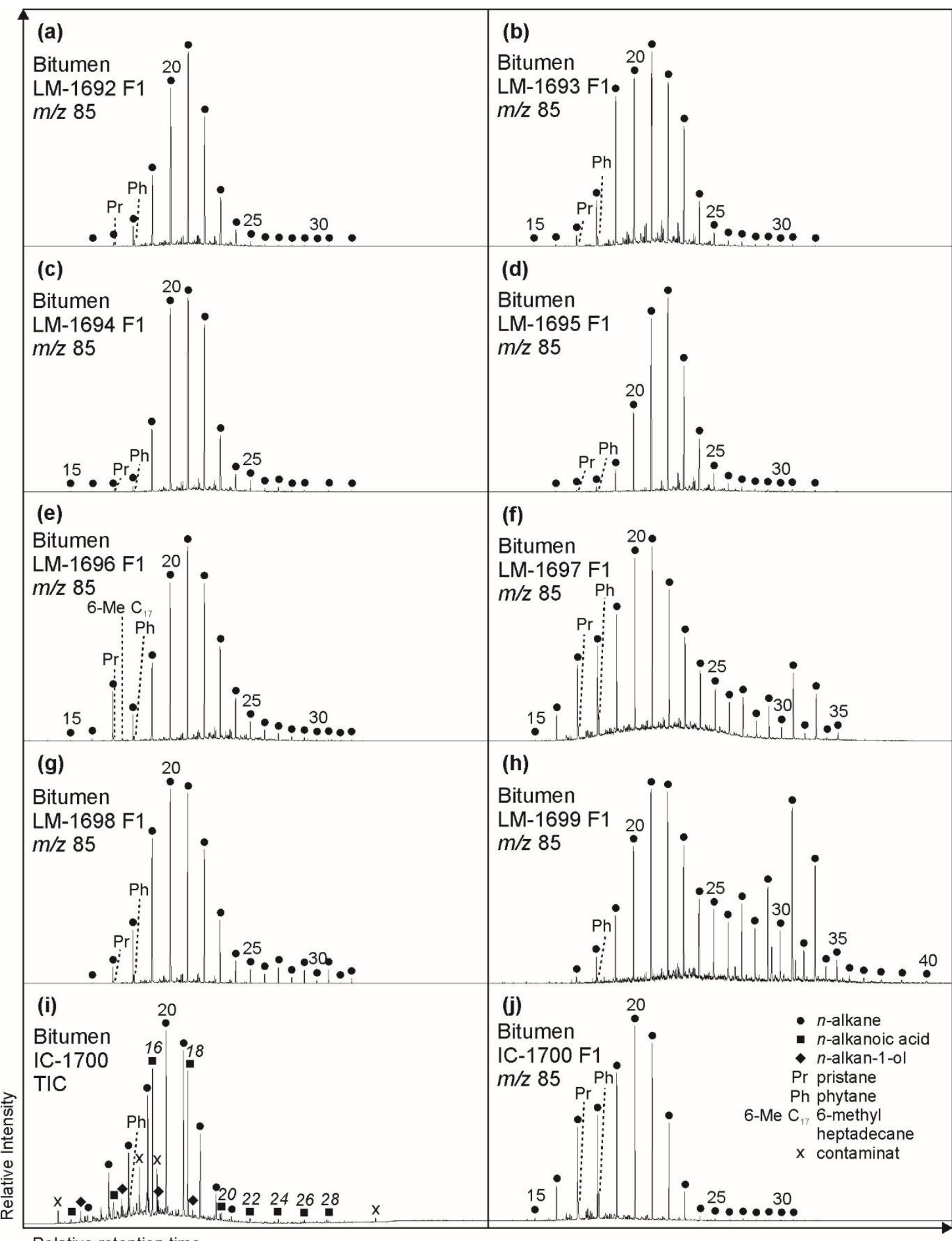

Relative retention time 
Figure S1. Partial GC-MS ion chromatograms $(\mathrm{m} / \mathrm{z}, 85 ; 10-60 \mathrm{~min})$ of the hydrocarbon fractions (F1) from bitumens of the Magadi cherts (LM-1692-1699; a-h). A total GC-MS ion chromatogram (TIC; 10-60 min) and a partial GC-MS ion chromatogram (m/z 85; 10-60 min) of the Great Geysir reference sinter (IC-1700) is shown in (i) and (j), respectively. A narrow bell-shaped $n$-alkane distribution in the mid-chain range (around $n-C_{21}$ ) is visible in all samples analyzed.

5 Additionally, odd-numbered long-chain $n$-alkanes are abundant in bitumens from most of the Green Bed cherts (LM-16971699; f-h). Notably, 6-methyl heptadecane appears in LM-1696 (e).

10 


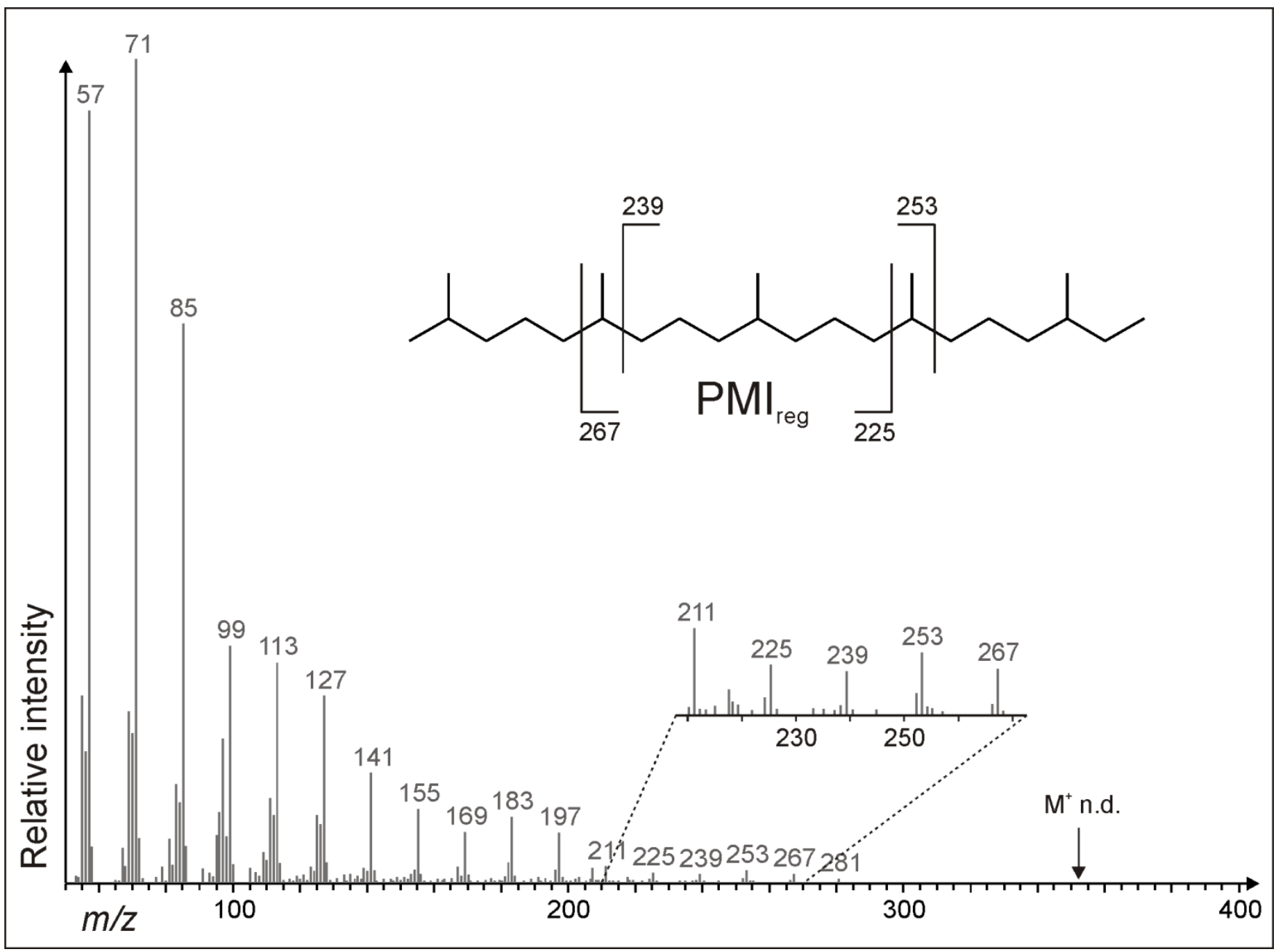

Figure S2. Mass spectrum of the regular $\mathrm{C}_{25}$ isoprenoid 2,6,10,14,18-pentamethylicosane (PMI $\mathrm{Peg}_{\mathrm{rg}}$ ) from kerogen of LM1693 (similar in kerogen pyrolysates from LM-1692 and LM-1695). Typical for this $\mathrm{C}_{25}$ isoprenoid isomer is the higher abundance of the fragments at 225 and $253 \mathrm{u}$, as compared to 239 and $267 \mathrm{u}$ (Risatti et al., 1984; Greenwood and Summons, 5 2003).The molecular ion $\left(\mathrm{M}^{+}\right)$at $352 \mathrm{u}$ was not detected (n.d.). 
Table S1. Mean $\delta^{13} \mathrm{C}_{\mathrm{V}-\mathrm{PDB}}$ values in \%o of fatty acids from bitumens

\begin{tabular}{|c|c|c|c|c|c|c|c|c|c|c|c|c|c|c|c|c|c|c|}
\hline & $\begin{array}{l}\text { LM- } \\
1692\end{array}$ & & $\begin{array}{l}\text { LM- } \\
1693\end{array}$ & & $\begin{array}{l}\text { LM- } \\
1694\end{array}$ & & $\begin{array}{l}\text { LM- } \\
1695\end{array}$ & & $\begin{array}{l}\text { LM- } \\
1696\end{array}$ & & $\begin{array}{c}\text { LM- } \\
1697\end{array}$ & & $\begin{array}{c}\text { LM- } \\
1698\end{array}$ & & $\begin{array}{c}\text { LM- } \\
1699\end{array}$ & & $\begin{array}{c}\text { IC- } \\
1700\end{array}$ & \\
\hline & 1692 & & 1693 & & 1694 & & 1695 & & & & & & & & & & & \\
\hline & Mean & \pm & Mean & \pm & Mean & \pm & Mean & \pm & Mean & \pm & Mean & \pm & Mean & \pm & Mean & \pm & Mean & \pm \\
\hline $\mathrm{C}_{12: 0}$ & -25.7 & 0.1 & -25.0 & 0.1 & & & & & & & & & & & & & -26.5 & 0.1 \\
\hline $\mathrm{C}_{13: 0}$ & & & -26.1 & 0.5 & & & & & & & & & & & & & -21.2 & 0.2 \\
\hline $\mathrm{C}_{14: 0}$ & -26.1 & $<0.1$ & -27.5 & 0.1 & -27.4 & $<0.1$ & -32.2 & 0.1 & & & -28.5 & 0.8 & & & & & -26.4 & 0.2 \\
\hline $\begin{array}{c}i-\mathrm{C}_{15: 0} \\
\text { ai- }\end{array}$ & & & -25.3 & $<0.1$ & -27.3 & 0.1 & & & & & & & & & & & -18.2 & $<0.1$ \\
\hline $\mathrm{C}_{15: 0}$ & -30.9 & $<0.1$ & -28.8 & 0.1 & -29.5 & 0.1 & & & & & & & & & & & -21.2 & 0.7 \\
\hline $\mathrm{C}_{15: 0}$ & -27.8 & 0.1 & -28.0 & 0.2 & -26.1 & 0.1 & -31.4 & 0.2 & & & -21.5 & 0.4 & -29.2 & $<0.1$ & -23.9 & 0.1 & -24.8 & 0.2 \\
\hline $\mathrm{C}_{16: 1}$ & & & & & & & & & & & & & -26.9 & 0.5 & & & & \\
\hline $\mathrm{C}_{16: 1}$ & & & & & & & & & & & & & -23.7 & 0.5 & & & & \\
\hline $\begin{array}{c}\mathrm{C}_{16: 0} \\
\text { ai- }\end{array}$ & -28.3 & $<0.1$ & -25.3 & $<0.1$ & -26.4 & $<0.1$ & -23.9 & 0.1 & -27.0 & 0.5 & -27.5 & 0.1 & -23.9 & $<0.1$ & -25.3 & 0.1 & -26.1 & $<0.1$ \\
\hline $\mathrm{C}_{17: 0}$ & -34.0 & $<0.1$ & -30.0 & 0.3 & -25.7 & 0.1 & & & & & & & & & & & & \\
\hline $\mathrm{C}_{17: 0}$ & -29.4 & 0.2 & -27.3 & 0.2 & -24.8 & 0.7 & -27.3 & $<0.1$ & -18.8 & 0.1 & -22.8 & 0.1 & -24.1 & 0.9 & -30.5 & 0.5 & -24.2 & 0.2 \\
\hline $\mathrm{C}_{18: 2}$ & & & -27.8 & 1.0 & -18.3 & 0.7 & & & -35.2 & 0.2 & & & -27.5 & 0.2 & & & & \\
\hline $\mathrm{C}_{18: 1}$ & -29.1 & 0.1 & -26.5 & 0.1 & -25.8 & $<0.1$ & & & -26.9 & $<0.1$ & -28.1 & 0.5 & -23.2 & 0.2 & -30.2 & 0.1 & & \\
\hline $\mathrm{C}_{18: 1}$ & -21.7 & 0.2 & -22.5 & 0.1 & & & & & & & & & -21.2 & 0.2 & & & & \\
\hline $\mathrm{C}_{18: 0}$ & -28.3 & 0.2 & -26.3 & 0.1 & -29.1 & 0.1 & -29.6 & 0.1 & -26.2 & 0.4 & -28.7 & 0.1 & -25.1 & 0.2 & -28.4 & 0.7 & -27.2 & 0.1 \\
\hline $\mathrm{C}_{19: 0}$ & & & & & & & & & & & -22.1 & 0.1 & -32.0 & 0.1 & & & -27.9 & $<0.1$ \\
\hline $\mathrm{C}_{20: 0}$ & -28.8 & 0.6 & -28.2 & $<0.1$ & -27.2 & $<0.1$ & & & -20.2 & 0.1 & -20.8 & 0.1 & -27.3 & 0.7 & -27.5 & 0.2 & -21.1 & 0.1 \\
\hline $\mathrm{C}_{21: 0}$ & & & & & & & & & & & -21.3 & $<0.1$ & -30.9 & 0.3 & & & & \\
\hline $\mathrm{C}_{22: 0}$ & & & & & & & & & -30.5 & 0.3 & -24.3 & 0.1 & -28.0 & 0.7 & -26.7 & $<0.1$ & -33.7 & 0.3 \\
\hline $\mathrm{C}_{23: 0}$ & & & & & & & & & & & -25.7 & $<0.1$ & -30.8 & 0.1 & & & -30.6 & $<0.1$ \\
\hline $\mathrm{C}_{24: 0}$ & -24.5 & 0.1 & -28.0 & 0.1 & -22.2 & 0.1 & -28.9 & 0.1 & -28.5 & 0.3 & -26.3 & 0.1 & -27.5 & 0.1 & -28.8 & $<0.1$ & -29.5 & 0.1 \\
\hline $\begin{array}{c}i-\mathrm{C}_{25: 0} \\
a i- \\
\mathrm{C}_{25: 0}\end{array}$ & & & $\begin{array}{l}-24.8 \\
-27.1\end{array}$ & $\begin{array}{l}0.2 \\
0.2\end{array}$ & & & & & & & & & & & & & & \\
\hline $\mathrm{C}_{25: 0}$ & & & -22.5 & 0.2 & & & & & & & -26.8 & 0.1 & -24.7 & 0.7 & & & -33.8 & 0.1 \\
\hline $\mathrm{C}_{26: 0}$ & -25.8 & 0.2 & -30.6 & $<0.1$ & -30.4 & 0.4 & & & -16.3 & 0.2 & -24.6 & 0.2 & -30.0 & 0.3 & -30.5 & 0.3 & -35.4 & 0.1 \\
\hline $\mathrm{C}_{27: 0}$ & & & & & & & & & & & -22.5 & 0.2 & & & & & -31.9 & 0.2 \\
\hline $\mathrm{C}_{28: 0}$ & & & & & & & & & & & -25.4 & $<0.1$ & & & & & -32.0 & $<0.1$ \\
\hline
\end{tabular}


Table S2. Mean $\delta^{13} \mathrm{C}_{\mathrm{V} \text {-PDB }}$ values in \%o of alcohols, ketones, mono- and diethers from bitumens

\begin{tabular}{|c|c|c|c|c|c|c|c|c|c|c|c|c|c|c|c|c|c|c|}
\hline & $\begin{array}{c}\text { LM- } \\
1692\end{array}$ & & $\begin{array}{c}\text { LM- } \\
1693\end{array}$ & & $\begin{array}{c}\text { LM- } \\
1694\end{array}$ & & $\begin{array}{c}\text { LM- } \\
1695\end{array}$ & & $\begin{array}{c}\text { LM- } \\
1696\end{array}$ & & $\begin{array}{c}\text { LM- } \\
1697\end{array}$ & & $\begin{array}{c}\text { LM- } \\
1698\end{array}$ & & $\begin{array}{l}\text { LM- } \\
1699\end{array}$ & & $\begin{array}{c}\text { IC- } \\
1700\end{array}$ & \\
\hline & Mean & \pm & Mean & \pm & Mean & \pm & Mean & \pm & Mean & \pm & Mean & \pm & Mean & \pm & Mean & \pm & Mean & \pm \\
\hline \multicolumn{19}{|c|}{ Alkan-1-ols } \\
\hline $\mathrm{C}_{12}$ & & & & & & & & & & & & & & & & & -29.9 & 0.4 \\
\hline $\mathrm{C}_{13}$ & & & & & & & & & & & & & & & & & -25.2 & 0.1 \\
\hline $\mathrm{C}_{14}$ & -28.5 & 0.2 & -39.5 & 0.1 & -37.3 & 0.1 & -19.6 & 0.5 & -27.1 & 0.7 & & & -19.4 & 0.2 & & & -30.2 & 0.1 \\
\hline $\mathrm{C}_{15}$ & & & & & & & & & & & & & & & & & -27.3 & $<0.1$ \\
\hline $\mathrm{C}_{16}$ & -34.8 & 0.2 & -25.1 & $<0.1$ & -35.5 & 0.4 & -36.0 & 0.3 & -35.9 & 0.2 & -33.1 & 0.2 & -33.6 & 0.4 & -30.9 & 0.3 & -24.4 & 0.1 \\
\hline $\mathrm{C}_{18}$ & -37.2 & 0.3 & -31.9 & 0.2 & -35.0 & 0.3 & -35.9 & 0.4 & -34.8 & 0.1 & -33.2 & $<0.1$ & -35.1 & 0.4 & -32.8 & 0.4 & -29.4 & 0.3 \\
\hline $\mathrm{C}_{20}$ & -30.9 & 0.1 & -29.9 & 0.6 & -32.7 & 0.1 & -30.3 & 0.2 & -29.8 & 0.1 & -24.8 & 0.2 & -29.5 & 0.1 & -30.9 & 0.1 & & \\
\hline $\mathrm{C}_{21}$ & & & & & & & -33.3 & 0.2 & & & & & & & & & & \\
\hline $\mathrm{C}_{22}$ & -29.6 & 0.4 & -32.4 & 0.3 & -28.7 & 0.1 & -30.3 & 0.2 & -25.2 & 0.4 & -34.6 & 0.2 & -26.7 & 0.3 & -31.5 & 0.3 & -25.9 & 0.2 \\
\hline $\mathrm{C}_{23}$ & & & & & & & & & & & & & -31.1 & 0.1 & -20.2 & 0.4 & & \\
\hline $\mathrm{C}_{24}$ & -20.2 & 0.3 & -36.5 & 0.1 & -28.3 & 0.3 & -32.3 & 0.6 & -30.1 & 0.7 & -22.6 & 0.8 & -25.0 & 0.2 & -30.0 & 0.3 & -24.0 & 0.1 \\
\hline $\mathrm{C}_{25}$ & & & & & & & & & & & & & -17.5 & 0.2 & -25.3 & 0.2 & & \\
\hline $\mathrm{C}_{26}$ & -32.1 & 0.5 & -33.8 & 0.7 & -17.4 & 0.2 & -28.5 & 0.9 & -26.5 & 0.3 & -29.2 & 0.1 & -21.7 & 0.3 & -21.8 & 0.2 & -27.7 & 0.5 \\
\hline $\mathrm{C}_{27}$ & & & & & & & & & & & & & -26.4 & 0.3 & & & & \\
\hline $\mathrm{C}_{28}$ & -21.3 & 1.0 & -26.7 & 0.5 & -20.6 & 0.4 & -26.6 & 1.0 & -18.7 & 0.3 & -22.8 & 0.1 & -25.4 & $<0.1$ & -23.5 & 0.1 & -23.0 & 0.2 \\
\hline $\mathrm{C}_{30}$ & & & & & -14.6 & 0.3 & & & & & -20.3 & 0.7 & & & -19.6 & 0.1 & -29.5 & 0.5 \\
\hline $\mathrm{C}_{32}$ & -26.4 & 0.7 & & & & & & & & & & & & & & & & \\
\hline
\end{tabular}

Glycerol mono- and diethers

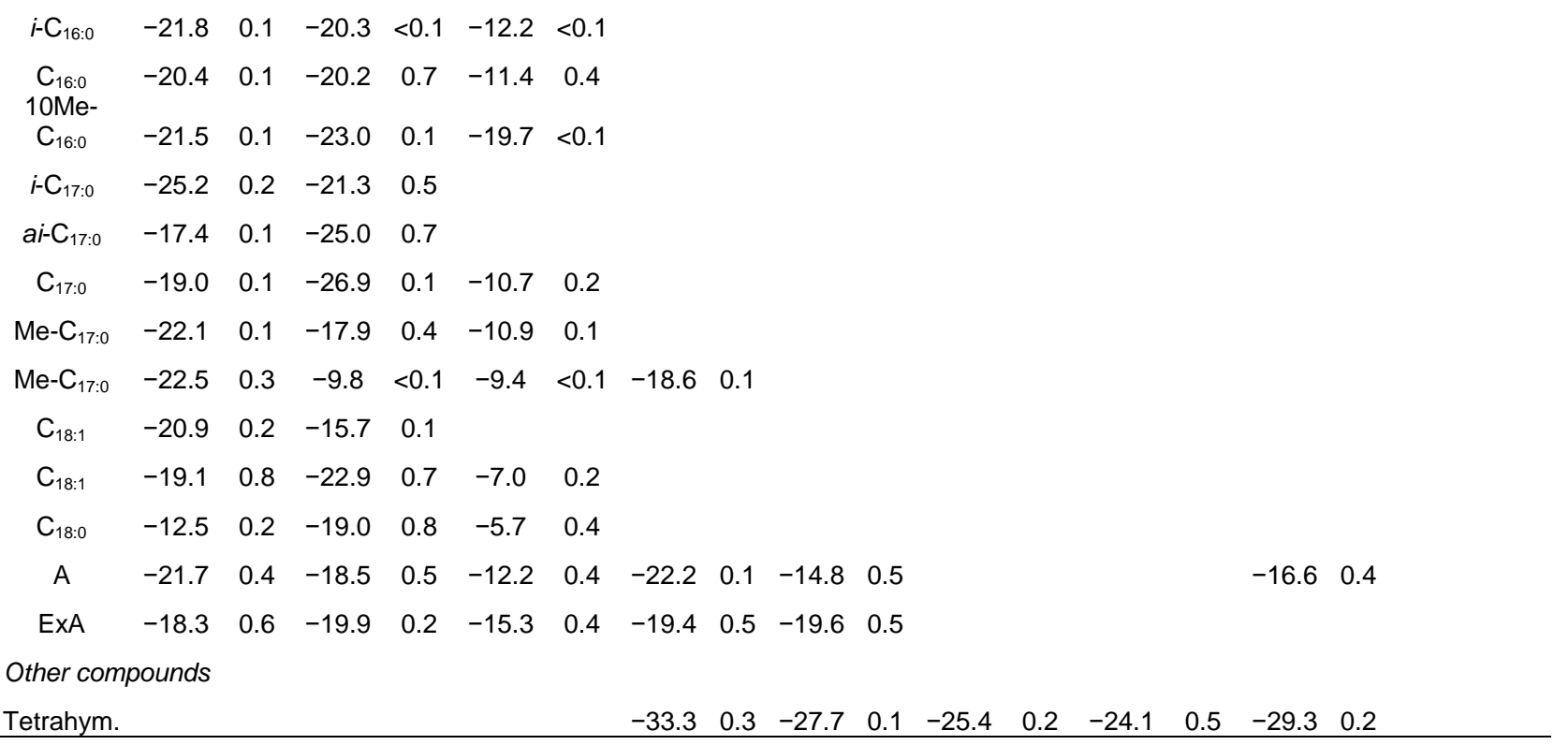


Table S3. Mean $\delta^{13} \mathrm{C}_{\mathrm{V}-\mathrm{PDB}}$ values in \%o of alkanes and isoprenoids from bitumens

\begin{tabular}{|c|c|c|c|c|c|c|c|c|c|c|c|c|c|c|c|c|c|c|}
\hline & LM- & & LM- & & LM- & & LM- & & LM- & & LM- & & LM- & & LM- & & IC- & \\
\hline & 1692 & & 1693 & & 1694 & & 1695 & & 1696 & & 1697 & & 1698 & & 1699 & & 1700 & \\
\hline & Mean & \pm & Mean & \pm & Mean & \pm & Mean & \pm & Mean & \pm & Mean & \pm & Mean & \pm & Mean & \pm & Mean & \pm \\
\hline$n-C_{16}$ & & & & & & & & & & & & & & & & & -37.1 & 0.1 \\
\hline$n-\mathrm{C}_{17}$ & & & -30.5 & 0.6 & & & & & -30.1 & 0.6 & -38.0 & 1.2 & & & & & -32.9 & $<0.1$ \\
\hline $6 \mathrm{Me}-\mathrm{C}_{17}$ & & & & & & & & & -30.3 & 0.2 & & & & & & & & \\
\hline$n-C_{18}$ & -32.5 & $<0.1$ & -32.1 & $<0.1$ & -31.8 & 0.5 & -35.6 & 0.5 & -33.8 & $<0.1$ & -34.4 & 0.1 & -34.3 & 0.1 & & & -33.4 & $<0.1$ \\
\hline$n-\mathrm{C}_{19}$ & -32.0 & 0.2 & -31.6 & 0.1 & -32.0 & 0.1 & -33.3 & 0.3 & -32.9 & 0.5 & -33.3 & 0.4 & -32.9 & $<0.1$ & -14.7 & 0.5 & -35.2 & 0.1 \\
\hline$i-\mathrm{C}_{20}$ & -31.1 & 0.1 & -30.0 & $<0.1$ & -34.1 & 0.3 & & & & & & & -29.8 & 0.2 & & & & \\
\hline$a i-\mathrm{C}_{20}$ & -32.1 & 0.8 & -33.2 & 0.1 & -29.0 & 0.8 & & & & & & & -27.5 & 0.1 & & & & \\
\hline$n-\mathrm{C}_{20}$ & -31.4 & $<0.1$ & -30.7 & 0.2 & -30.9 & 0.3 & -32.3 & 0.4 & -33.2 & $<0.1$ & -32.6 & 0.4 & -32.7 & 0.2 & -38.9 & 0.3 & -33.6 & $<0.1$ \\
\hline$i-\mathrm{C}_{21}$ & -32.3 & $<0.1$ & -31.2 & $<0.1$ & -32.7 & $<0.1$ & -30.9 & 0.1 & -34.4 & 0.7 & -35.3 & 0.5 & -33.2 & 0.3 & & & & \\
\hline$a i-C_{21}$ & -29.8 & $<0.1$ & -31.5 & $<0.1$ & -30.5 & 0.3 & -31.9 & 0.4 & -28.6 & 0.1 & -36.5 & 0.3 & -32.2 & 0.1 & & & & \\
\hline$n-\mathrm{C}_{21}$ & -31.6 & $<0.1$ & -31.0 & 0.2 & -31.2 & 0.4 & -31.9 & 0.1 & -32.9 & $<0.1$ & -31.6 & $<0.1$ & -33.6 & $<0.1$ & -35.0 & 0.7 & -34.0 & $<0.1$ \\
\hline$i-\mathrm{C}_{22}$ & -29.9 & 0.8 & -33.2 & 0.1 & -29.6 & 0.1 & -34.0 & 0.3 & -34.1 & 0.1 & -34.9 & 0.1 & -33.6 & 0.1 & & & & \\
\hline$a i-\mathrm{C}_{22}$ & -29.1 & 0.2 & -37.4 & 0.8 & -28.9 & 0.1 & -34.4 & 0.2 & -33.3 & $<0.1$ & -32.6 & 0.1 & -33.3 & 0.3 & & & & \\
\hline$n-C_{22}$ & -32.1 & 0.2 & -31.7 & $<0.1$ & -32.0 & 0.3 & -32.3 & 0.1 & -32.8 & $<0.1$ & -31.6 & 0.2 & -34.4 & 0.2 & -36.7 & 0.3 & -37.5 & $<0.1$ \\
\hline$i-\mathrm{C}_{23}$ & -33.8 & 0.1 & -30.3 & 0.1 & -29.1 & 0.1 & -32.1 & $<0.1$ & -31.4 & $<0.1$ & -36.5 & 0.1 & -31.3 & 0.2 & & & & \\
\hline$a i-\mathrm{C}_{23}$ & -33.7 & 0.2 & -27.3 & 0.1 & -28.8 & 0.1 & -33.1 & 0.1 & -32.1 & 0.1 & -39.1 & 0.4 & -30.2 & 0.4 & & & & \\
\hline$n-\mathrm{C}_{23}$ & -32.1 & $<0.1$ & -32.2 & 0.2 & -32.2 & $<0.1$ & -32.4 & 0.1 & -32.6 & 0.3 & -34.5 & $<0.1$ & -33.4 & 0.1 & -25.4 & 0.1 & -39.6 & 0.1 \\
\hline$i-\mathrm{C}_{24}$ & -31.8 & $<0.1$ & -33.9 & 0.1 & -32.5 & $<0.1$ & -29.8 & 0.4 & -35.2 & $<0.1$ & & & -36.3 & 0.3 & & & & \\
\hline$a i-\mathrm{C}_{24}$ & -26.2 & 0.5 & -32.6 & 0.2 & -31.5 & 0.1 & -29.0 & 0.1 & -34.3 & 0.3 & & & -29.5 & 0.7 & & & & \\
\hline$n-C_{24}$ & -32.7 & 0.2 & -33.7 & $<0.1$ & -31.6 & $<0.1$ & -31.8 & 0.2 & -32.5 & 0.5 & -30.6 & 0.2 & -32.2 & 0.1 & -27.6 & 0.2 & -39.2 & $<0.1$ \\
\hline$i-\mathrm{C}_{25}$ & -30.8 & $<0.1$ & -31.2 & 0.4 & -25.3 & $<0.1$ & -34.6 & 0.1 & -28.4 & 0.4 & & & & & & & & \\
\hline$a i-C_{25}$ & -26.8 & 0.4 & -35.9 & 0.4 & -22.6 & 0.2 & -30.4 & 0.7 & -28.0 & 0.2 & & & & & & & & \\
\hline$n-C_{25}$ & & & & & & & -32.0 & $<0.1$ & -30.2 & 0.1 & -34.7 & 0.1 & -34.9 & 0.2 & -25.6 & 0.1 & & \\
\hline$n-C_{26}$ & & & & & & & -29.8 & 0.1 & -29.9 & 0.2 & -34.0 & 0.2 & -25.8 & $<0.1$ & -34.8 & 0.3 & & \\
\hline$n-\mathrm{C}_{27}$ & & & & & & & & & & & -36.3 & 0.2 & -23.7 & 0.1 & -30.8 & 0.1 & & \\
\hline$n-\mathrm{C}_{28}$ & & & & & & & & & & & -33.8 & 0.2 & -24.2 & 0.3 & -27.9 & 0.1 & & \\
\hline$n-\mathrm{C}_{29}$ & & & & & & & & & & & -28.8 & 0.4 & -23.9 & 0.5 & -21.5 & 0.2 & & \\
\hline$n-C_{30}$ & & & & & & & & & & & & & & & -26.9 & 0.1 & & \\
\hline$n-C_{31}$ & & & & & & & & & & & -20.9 & 0.6 & -24.4 & 0.1 & -21.1 & 0.4 & & \\
\hline$n-C_{32}$ & & & & & & & & & & & & & & & -24.5 & 0.2 & & \\
\hline$n-\mathrm{C}_{33}$ & & & & & & & & & & & & & & & -25.8 & $<0.1$ & & \\
\hline \multicolumn{19}{|c|}{ Isoprenoids } \\
\hline $\operatorname{Pr}$ & & & -31.7 & 0.1 & & & & & -30.2 & 0.1 & -32.2 & 0.2 & -35.8 & 0.1 & & & -34.5 & 0.2 \\
\hline $\mathrm{Ph}$ & -33.3 & $<0.1$ & -30.9 & 0.4 & -30.0 & 0.6 & -36.1 & 1.2 & -34.7 & 0.1 & -33.8 & 0.1 & -35.3 & $<0.1$ & & & -38.6 & 0.1 \\
\hline
\end{tabular}


Table S4. Mean $\delta^{13} \mathrm{C}_{\mathrm{V}-\mathrm{PDB}}$ values in \%o of alkanes and isoprenoids from kerogens

\begin{tabular}{|c|c|c|c|c|c|c|c|c|c|c|c|c|}
\hline & LM- & & LM- & & LM- & & LM- & & LM- & & LM- & \\
\hline & 1692 & & 1693 & & 1694 & & 1695 & & 1697 & & 1698 & \\
\hline & Mean & \pm & Mean & \pm & Mean & \pm & Mean & \pm & Mean & \pm & Mean & \pm \\
\hline$n-C_{15}$ & & & & & & & -30.5 & 0.6 & & & & \\
\hline$n-\mathrm{C}_{16}$ & & & & & & & -30.4 & 0.1 & & & -30.9 & 0.1 \\
\hline$n-\mathrm{C}_{17}$ & & & & & & & -30.3 & $<0.1$ & -26.0 & 0.3 & -31.3 & $<0.1$ \\
\hline$n-C_{18}$ & -36.3 & 0.7 & -35.0 & 0.9 & & & -31.5 & 0.2 & -25.4 & 0.4 & -34.9 & $<0.1$ \\
\hline$n-C_{19}$ & -32.0 & 0.3 & -29.2 & 0.7 & & & -27.2 & 0.1 & -24.0 & $<0.1$ & -34.6 & 0.1 \\
\hline$n-\mathrm{C}_{20}$ & -27.8 & 0.6 & -33.7 & 0.8 & -34.6 & 0.6 & -26.9 & 0.1 & -22.1 & $<0.1$ & -35.8 & 0.2 \\
\hline$n-C_{21}$ & -28.1 & 0.4 & -31.5 & 0.3 & -29.6 & 0.5 & -27.6 & 0.7 & -22.0 & 0.2 & -34.3 & 0.8 \\
\hline$n-\mathrm{C}_{22}$ & -31.1 & $<0.1$ & -30.9 & 0.2 & -26.6 & 0.1 & -28.8 & 0.1 & -23.0 & 0.9 & -32.9 & 0.1 \\
\hline$n-C_{23}$ & -31.8 & 0.1 & -29.3 & 0.4 & -21.0 & 0.1 & -27.3 & $<0.1$ & -22.4 & 0.1 & -34.5 & 0.8 \\
\hline$n-\mathrm{C}_{24}$ & -26.5 & 0.6 & -30.5 & 0.3 & -24.7 & 0.4 & -26.9 & 0.2 & -22.9 & 0.5 & -35.3 & 0.2 \\
\hline$n-\mathrm{C}_{25}$ & -28.7 & 0.5 & -30.9 & 1.0 & -22.7 & 0.2 & -27.3 & $<0.1$ & -24.4 & 0.3 & -34.9 & $<0.1$ \\
\hline$n-C_{26}$ & -26.6 & 0.5 & -27.4 & 0.1 & -23.3 & 0.2 & -25.4 & $<0.1$ & -24.1 & 0.1 & -29.7 & 0.1 \\
\hline$n-\mathrm{C}_{27}$ & -29.8 & 0.3 & -30.6 & 0.1 & -18.9 & 0.1 & -24.8 & $<0.1$ & -22.9 & 0.4 & -28.0 & 0.1 \\
\hline$n-\mathrm{C}_{28}$ & -30.4 & 0.4 & -28.8 & 0.7 & -24.3 & 0.4 & -23.6 & 0.3 & -22.6 & 0.3 & -28.0 & 0.1 \\
\hline$n-C_{29}$ & -25.9 & $<0.1$ & -29.1 & 0.8 & -16.8 & 1.0 & -25.8 & 0.2 & -22.8 & 0.2 & -26.3 & 0.3 \\
\hline$n-C_{30}$ & -29.6 & 0.6 & -32.3 & 0.4 & -22.0 & 0.8 & -24.3 & 0.3 & -22.1 & $<0.1$ & -26.0 & 0.1 \\
\hline$n-C_{31}$ & -24.7 & 0.2 & -31.0 & 0.4 & -23.0 & 0.4 & -23.5 & $<0.1$ & -22.4 & 0.1 & -27.1 & 0.6 \\
\hline$n-C_{32}$ & -26.7 & 0.4 & -31.3 & 1.0 & -21.9 & 0.6 & -22.6 & 0.4 & -21.5 & $<0.1$ & -24.1 & 0.6 \\
\hline$n-C_{33}$ & -24.8 & 0.7 & & & -26.0 & 0.3 & -23.6 & 0.2 & -21.4 & 0.4 & -26.2 & 0.2 \\
\hline$n-C_{34}$ & -27.6 & 0.1 & & & -24.6 & 1.0 & -23.2 & 0.1 & -21.2 & 0.1 & -25.4 & $<0.1$ \\
\hline$n-\mathrm{C}_{35}$ & -28.9 & 0.3 & & & -26.0 & 0.4 & -24.4 & 0.7 & -20.4 & 0.5 & -28.2 & 0.5 \\
\hline$n-\mathrm{C}_{36}$ & & & & & -26.1 & 0.2 & -27.4 & 0.7 & -21.0 & 0.1 & -23.7 & 0.4 \\
\hline$n-\mathrm{C}_{37}$ & & & & & & & -27.7 & 0.9 & -20.8 & 0.4 & -26.9 & 0.2 \\
\hline$n-\mathrm{C}_{38}$ & & & & & & & -25.6 & $<0.1$ & -19.3 & 0.6 & -25.3 & $<0.1$ \\
\hline$n-C_{39}$ & & & & & & & & & -21.8 & 0.1 & & \\
\hline$n-\mathrm{C}_{40}$ & & & & & & & & & -21.6 & 0.5 & & \\
\hline \multicolumn{13}{|c|}{ Isoprenoids } \\
\hline Far & & & & & & & -33.0 & 0.2 & & & & \\
\hline Nor & & & & & & & -35.3 & 0.1 & & & & \\
\hline $\operatorname{Pr}$ & & & & & & & -32.3 & 0.3 & & & & \\
\hline $\mathrm{Ph}$ & -25.1 & 0.3 & -26.8 & 0.2 & & & -28.5 & $<0.1$ & & & & \\
\hline $\mathrm{PMI}_{\text {reg }}$ & -22.0 & 0.3 & -24.0 & 0.4 & -14.5 & $<0.1$ & -24.6 & 0.1 & & & & \\
\hline
\end{tabular}


Table S5. Coefficients of determination $\left(\mathrm{R}^{2}\right)$ for Raman fittings

\begin{tabular}{lrrrrrrr}
\hline & LM-1692 & LM-1693 & LM-1694 & LM-1695 & LM-1696 & LM-1697 & LM-1698 \\
\hline Flake 1 & 0.995 & 0.996 & 0.995 & 0.975 & 0.985 & 0.984 & 0.989 \\
Flake 2 & 0.996 & 0.997 & 0.995 & 0.989 & 0.992 & 0.987 & 0.975 \\
Flake 3 & 0.994 & 0.998 & 0.992 & 0.982 & 0.989 & 0.987 & 0.984 \\
Flake 4 & 0.988 & 0.992 & 0.994 & 0.986 & 0.994 & 0.987 & 0.991 \\
Flake 5 & 0.996 & 0.997 & 0.989 & 0.992 & 0.997 & 0.984 & 0.986 \\
Flake 6 & 0.996 & 0.997 & 0.994 & 0.974 & 0.989 & 0.986 & 0.985 \\
Flake 7 & 0.996 & 0.997 & 0.995 & 0.954 & 0.992 & 0.979 & 0.986 \\
Flake 8 & 0.995 & 0.998 & 0.979 & 0.992 & 0.982 & 0.970 & 0.981 \\
Flake 9 & 0.997 & 0.998 & 0.988 & 0.987 & 0.992 & 0.985 & 0.986 \\
Flake 10 & 0.998 & 0.995 & 0.991 & 0.991 & 0.989 & 0.987 & 0.992 \\
Mean & 0.995 & 0.996 & 0.991 & 0.982 & 0.990 & 0.984 & 0.986 \\
\hline
\end{tabular}

5

10

15 
Table S6. Mean concentrations of selected $n$-alkanes, archaeol (A) and extended archaeol (ExA) in the interior and exterior of LM-1692 and LM-1695 (mg/g TOC).

\begin{tabular}{|c|c|c|c|c|c|c|c|c|c|c|}
\hline & $n-C_{18}$ & $n-C_{19}$ & $n-C_{20}$ & $n-C_{21}$ & $n-C_{22}$ & $n-C_{23}$ & $n-C_{24}$ & $n-C_{25}$ & A & ExA \\
\hline 1692 exterior & 0.02 & 0.11 & 0.13 & 0.18 & 0.13 & 0.16 & 0.03 & 0.02 & 0.21 & 0.12 \\
\hline 1692 interior & 0.02 & 0.09 & 0.12 & 0.22 & 0.20 & 0.16 & 0.04 & 0.03 & 0.17 & 0.12 \\
\hline 1695 exterior & 0.08 & 0.35 & 0.42 & 1.20 & 0.96 & 0.74 & 0.42 & 0.25 & 0.24 & 0.09 \\
\hline 1695 interior & 0.07 & 0.32 & 0.49 & 1.83 & 1.33 & 0.76 & 0.32 & 0.13 & 0.42 & 0.16 \\
\hline
\end{tabular}

